\title{
Herbarium für zu Hause
}

Eine Schritt-für-Schritt-Anleitung. Doris Grappendorf

Sie möchten die heimischen Heilpflanzen besser kennenlernen? Ein Herbarium ist hierfür eine wunderbare Möglichkeit. Sie könnten zum Beispiel bei einer Heilpflanzenexkursion von jeder vorgestellten Heilpflanze (in Rücksprache mit der Exkursionsleitung) eine pflücken, einen Zettel mit dem Namen der Pflanze anheften, die Pflanze dann zu Hause pressen, trocknen und auf eine Karte kleben. Schon hätten Sie Ihr erstes kleines Herbarium in der Hand. Nun können Sie sich die Heilpflanze immer wieder betrachten und in Erinnerung rufen. Sie können die Pflanzenkarte auch

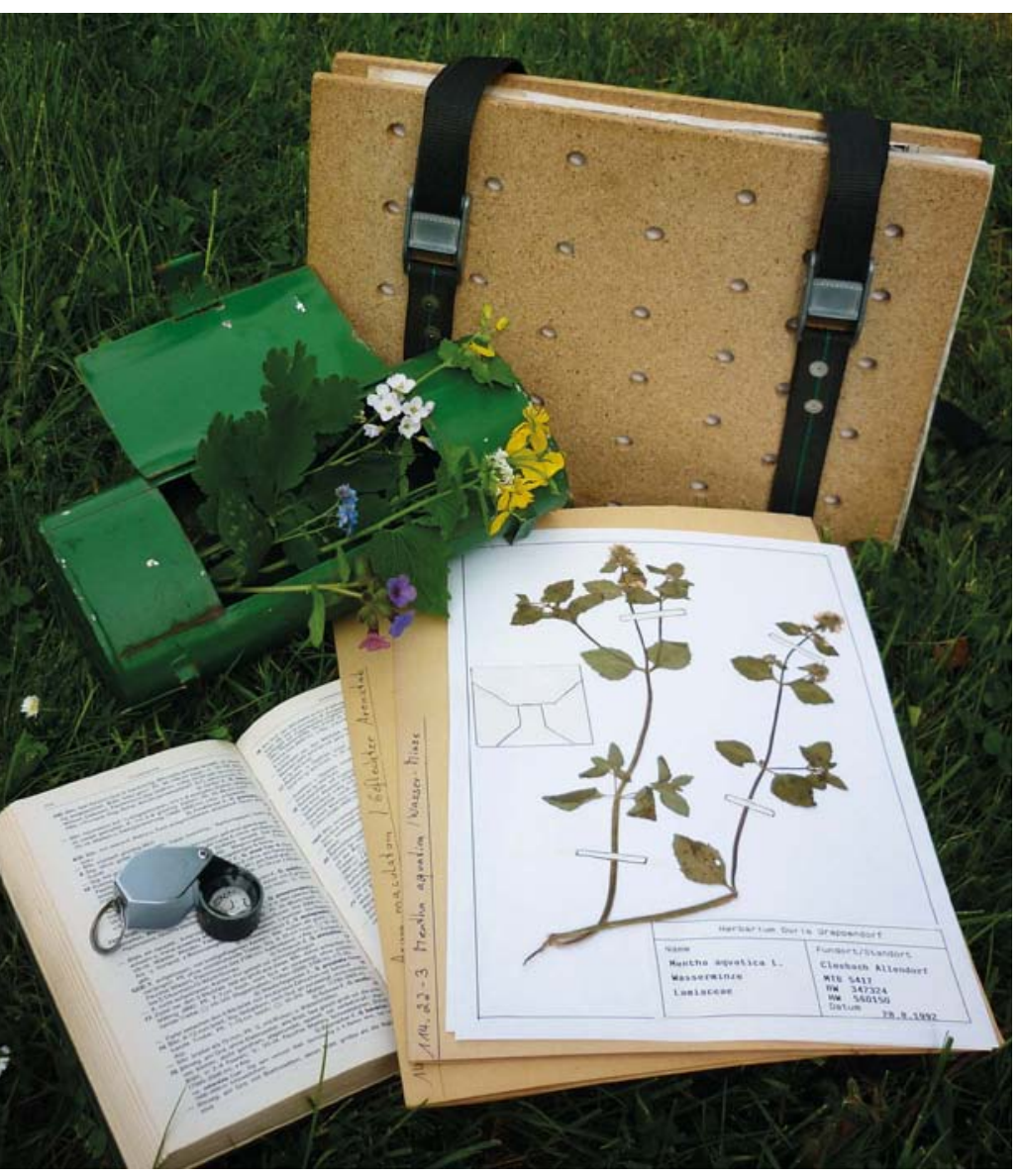

Abb. 1 Herbarbögen mit gepressten und getrockneten Heilpflanzen eignen sich nicht nur zum Erinnern und Lernen, sie bieten auch einen wertvollen Vergleich für neu gefundene Pflanzen. Quelle: Doris Grappendorf mit einem kleinen Text über die Heilwirkung versehen. Wenn Sie bei Ihrem nächsten Spaziergang eine Pflanze finden, von der Sie denken, sie sei der vorgestellten Heilpflanze ähnlich, können Sie diese mit Ihrer Pflanzenkarte - man nennt sie Herbarbeleg - vergleichen. Kein Foto hält dieser naturgetreuen Abbildung der gepressten Heilpflanze stand.

Auf diese Weise habe ich vor fast vierzig Jahren begonnen, die heimische Pflanzenwelt kennenzulernen. Mein Herbarium umfasst mittlerweile über zweitausend Pflanzenbelege. Vor allem im Winter kann es eine Wohltat sein, sich diese getrockneten Pflanzen wie in einem Fotoalbum anzuschauen und sich an die schönen Momente in der Natur zu erinnern, in denen man sie entdeckt hat. Nachfolgend lernen Sie nach einem kurzen geschichtlichen Abriss kennen, wie Sie ein solches Herbarium Schritt für Schritt anlegen können.

\section{Was ist ein Herbarium?}

Mit dem Begriff „Herbarium“ bezeichnet man eine Sammlung gepresster und getrockneter Pflanzen, die auf Papierbögen geklebt sind. Da die Pflanzen durch das Herbarisieren hervorragend erhalten bleiben, sind sie vor allem für vergleichende Studien gut geeignet. So hat diese Methode unter den Botanikern eine lange Tradition.

\section{Seit wann gibt es Herbarien?}

Das älteste bekannte Herbarium hat Luca Ghini, Professor der Botanik an der Universität Pisa in Italien, erstellt. Leider existiert sein Herbarium nicht mehr. Jedoch ist das Herbarium seines Schülers U. Adrovandi bis heute erhalten geblieben. Es enthält 4368 Pflanzen und wird in der Universität Bologna in Italien aufbewahrt. Zu dieser Zeit entstand auch das älteste bekannte deutsche Herbarium durch Caspar Ratzenberger. Seine Entstehung wird mit dem Jahr 1559 angegeben. Es enthält 746 Pflanzen.

Damals war es üblich, Sammlungen gepresster und getrockneter Pflanzen wie Bücher zu binden, die man Kräuterbücher nannte. Im Kasseler Naturkundemuseum kann man solche 460 Jahre alten Bücher besichtigen.

Fast jede höhere Schule besaß früher für Studienzwecke ein Herbarium, und die Studierenden mussten ein solches anlegen. Auch jeder Apotheker hatte ein mehr oder weniger umfangreiches Herbarium. Goethe besaß ebenfalls eines mit 1800 Pflanzenbelegen. 
Der Begriff Herbarium wurde erst 200 Jahre später durch Carl von Linné geprägt. Der Naturforscher wurde unter anderem durch seine 1735 veröffentlichte Pflanzensystematik und der darauf begründeten zweiteiligen botanischen Bezeichnung von Pflanzen (Gattung und Art) weltbekannt. Linné ließ die Herbarbögen nicht mehr zu Büchern binden, sondern bewahrte sie einzeln auf. So konnte er neu hinzukommende Pflanzen in das von ihm erstellte System einsortieren. Er nannte diese Pflanzenkarten Herbarbelege. Durch seine große Aktivität und Bekanntheit verbreitete sich diese Technik sehr schnell und wird heute überall verwendet.

Auf der Welt gibt es derzeit 2721 öffentliche Herbarien in 180 Ländern mit insgesamt 361 Millionen Herbarbelegen. Das kann man dem „Index Herbariorum“ entnehmen. Das ist ein Register aller Pflanzensammlungen, das regelmäßig veröffentlicht wird. Das größte Herbarium befindet sich in The Royal Botanic Gardens (Kew) in der Nähe von London und enthält etwa 7 Millionen Belege. In Deutschland gibt es 34 öffentliche Herbarien mit insgesamt etwa 15 Millionen Belegen. Davon befindet sich in Berlin mit 3,6 Millionen Pflanzenbelegen die größte Sammlung Deutschlands und auch eine der größten der Welt.

Für ein eigenes langlebiges Herbarium ist im ersten Schritt das richtige Sammeln der Pflanzen ausschlaggebend, bevor die Pflanzen gepresst, getrocknet und schließlich auf Herbarbögen geklebt werden können.

\section{Schritt 1: Pflanzen sammeln}

Für das Sammeln der Pflanzen, die Teil des eigenen Herbariums werden sollen, eignen sich am besten Frühstücksbeutel. In ihnen halten sich die frischen Pflanzen gut. Auf einem Zettel oder Klebeetikett kann man den Namen der Pflanze notieren und dazulegen.

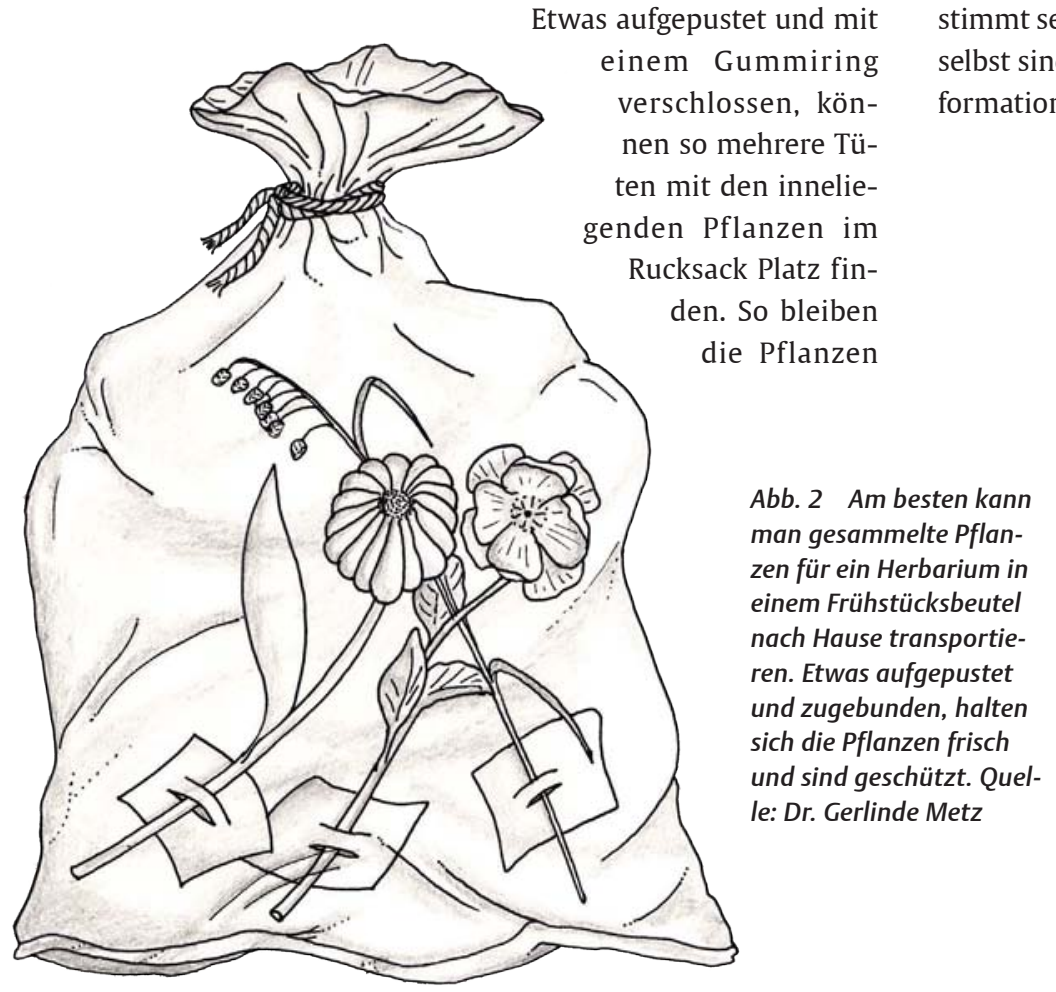

nahezu unversehrt und können zu Hause in Ruhe bestimmt oder gleich in die Presse gelegt werden.

Wenn man schon weiß, dass man nach der Exkursion keine Zeit haben wird, die Pflanzen in die Presse zu legen, kann eine Sammelmappe hilfreich sein. Sie sieht ähnlich aus wie eine einfache Presse und besteht aus zwei Pappen. Diese haben etwa die Größe der späteren Herbarbögen (siehe Abschnitt „Schritt 4: Pflanzen aufkleben“). Zwischen den Pappen sind mehrere Einlegebögen aus zum Beispiel Zeitungspapier, einer für jede gesammelte Pflanze (siehe Abschnitt „Pflanzen pressen und trocknen“). Mit zwei Riemen, Gürteln oder starken Gummibändern wird die Sammelmappe fest zusammengehalten.

Die ausgewählte Pflanze sollte gut entwickelt, nicht verkrüppelt oder von Insekten angefressen sein. Wenn möglich, sollte sie Blüte und Samen tragen. Auch die Grundblätter gehören dazu. Die Pflanze sollte so komplett wie möglich sein, wenn ihre Größe dies zulässt. Bevor man die Pflanze in die Tüte steckt, notiert man alle Daten, die später an der gepressten Pflanze nicht mehr nachvollzogen werden können. Dabei ist die Angabe des Datums und Fundorts wichtiger als der Pflanzenname selbst. Die getrocknete Pflanze kann jederzeit nachbestimmt werden, nicht jedoch Datum und Fundort.

Den Fundort sollte man genau dokumentieren. Man sollte ihn so genau beschreiben, dass ein Dritter den Ort nach der Beschreibung wiederfinden kann.

Es ist wichtig, so sorgfältig vorzugehen, denn es könnte sein, dass man sein Herbarium irgendwann einmal verschenken oder an eine wissenschaftliche Institution vererben möchte. Für andere Botaniker sind solche Pflanzensammlungen jedoch nur wertvoll, wenn sie mindestens diese beiden Angaben (Fundort und Datum) enthalten. Sie müssen nicht aufgeklebt und auch nicht bestimmt sein, denn all das kann nachgeholt werden. Auch für einen selbst sind die Angaben wichtig, damit man nach Jahren diese Inormationen nachvollziehen kann.

\section{HINTERGRUNDWISSEN}

\section{Geschützte Pflanzen}

Aus Naturschutzgebieten und Nationalparks dürfen keine Pflanzen oder Pflanzenteile entnommen werden. In anderen Gebieten dürfen nur die nicht geschützten Pflanzen gesammelt werden. Jedoch sollten auch jene Pflanzen geschont werden, deren Population nur wenige Individuen umfasst. 


\section{Schritt 2: Pflanzen bestimmen}

Wenn nicht schon vor Ort geschehen, bestimmt man die gesammelten Pflanzen zu Hause. Da es sehr viel einfacher ist, die ersten Bestimmungsversuche an frischen Pflanzen durchzuführen, sollte man die Pflanzen - vor allem zu Beginn - besser in Tüten und nicht in einer Sammelmappe mit nach Hause nehmen.

Sie können die Pflanzen nun mit einem illustrierten Buch oder mit einem wissenschaftlichen Bestimmungsbuch identifizieren. Für den Anfang ist ein illustriertes Buch empfehlenswert und einfacher zu handhaben (zum Beispiel: [3]). Darin sind die Pflanzen nach Blütenfarbe sortiert und innerhalb der Blütenfarbe nach Blütenformen. So hat man sehr schnell eine erste Orientierung.

Für Fortgeschrittene empfiehlt sich ein wissenschaftlicher Bestimmungsschlüssel (zum Beispiel: [4]). In solchen wissenschaftlichen Bestimmungsbüchern sind keine Fotos, sondern man muss Fragen beantworten, beispielsweise, ob die Blätter wechselständig oder gegenständig sind. Immer nur eine der vorgegebenen Antworten ist richtig, sodass man Schritt für Schritt ans Ziel gelangt.

Hat man die Pflanze erfolgreich bestimmt, werden der deutsche und der wissenschaftliche Name, sowie - falls noch nicht geschehen - das Datum und der Fundort auf einen Zettel übertragen. Dieser kommt dann zusammen mit der Pflanze in die Pflanzenpresse. Sollte es nicht gelingen, eine gefundene Pflanze sicher zu bestimmen, sollte man das Exemplar dennoch pressen und trocknen. Im getrockneten Zustand kann man sie nachbestimmen.

\section{Schritt 3: Pflanzen pressen und trocknen}

Nach dem Sammeln der Pflanzen sollten diese so schnell wie möglich in die Pflanzenpresse kommen. Zunächst wird die Pflanze hierfür in einen sogenannten Einlegebogen gelegt. Das ist ein Doppelbogen aus Zeitungspapier oder auch speziellem Löschpapier. Man breitet die Pflanze in möglichst natürlicher Position aus. Dies macht man mit der rechten Hand. Die linke Hand klappt von oben nach unten den Doppelbogen zu, während man die rechte Hand langsam herauszieht.

Durch diese Technik kommt die Pflanze weitgehend wie gewünscht zum Liegen. Je sorgfältiger das Ausbreiten der Pflanze geschieht, desto schöner und aussagekräftiger wird später die Pflanze in getrocknetem Zustand sein. Ist die Pflanze größer als der Bogen, knickt man sie vorsichtig, beispielsweise am Stängel zwischen Blattansätzen.

Wichtig ist, dass der Zettel mit dem Namen, der Angabe des Fundortes und des Datums mit in den Einlegebogen gegeben wird. Auf den so gefüllten Einlegebogen wird nun eine Zwischenlage aus Zeitungs- oder Löschpapier gelegt. Darauf kommt ein Bogen Wellpappe, sodass nach und nach ein Packen entsteht, der im Wechsel Wellpappe, Zwischenlage, Einlegebogen, Zwischenlage und Wellpappe enthält.

Hat man die Pflanzen unterwegs schon in die Sammelmappe eingelegt, sortiert man lediglich Zwischenlagen und Wellpappen zwischen die Einlegebögen.

Der ganze Stapel mit allen Pflanzen wird nun in die Presse eingelegt. Pflanzenpressen gibt es in verschiedenen Ausführungen. Für den Anfang sind auch einige dicke Bücher oder Steine geeig-

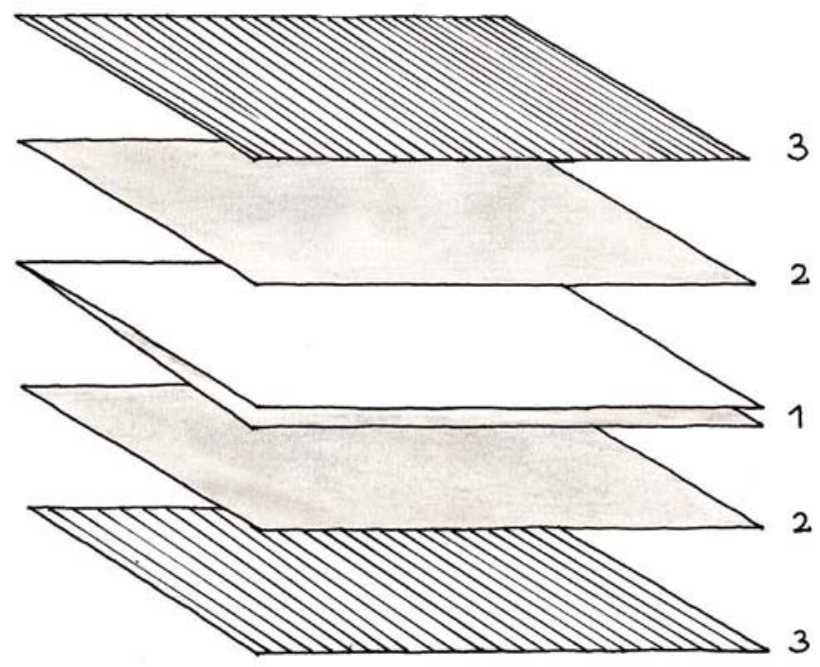

Abb. 3 Beim Trocknen und Pressen von Pflanzen in je einem Einlegebogen ist die richtige Reihenfolge der Zwischenlagen entscheidend: 1. Einlegebogen 2. Zwischenlage aus Zeitungs- oder Löschpapier 3. Wellpappe. Quelle: Dr. Gerlinde Metz

net, die Druck auf den Pflanzenstapel ausüben. Die einfachste Presse besteht aus zwei Sperrholzbrettern, um die zwei Gürtel oder Zurrgurte gezogen werden.

Wichtigstes Kriterium bei der Wahl einer Presse ist, dass die Pflanzen gut durchlüftet werden und möglichst schnell trocknen können. So behalten sie ihre Farbe und werden nicht schwarz oder schimmelig. Die Presse sollte an einem warmen oder sonnigen Ort stehen.

Wie schön die Pflanzen nach dem Pressen aussehen, hängt ganz davon ab, wie schnell sie in der Presse trocknen. Anfangs sollte man die Pflanzen deshalb täglich umlegen. Dazu ersetzt man die Zwischenlagen und Pappen durch trockene. Die Einlegebögen, in denen die Pflanzen liegen, wechselt man nicht, da die feuchten Pflanzen sehr empfindlich sind und nach Anheben oft nicht wieder in der gewünschten Lage ausgebreitet werden können. Nach täglichem Umlegen sind die meisten Pflanzen nach etwa einer Woche trocken. Sie sind dann steif, rascheln und fühlen sich nicht mehr kalt an. Die Einlegebögen mit den Pflanzen können anschließend in einer Mappe bis zum Einkleben aufbewahrt werden.

\section{Schritt 4: Pflanzen auf Herbarbögen kleben}

Es kann gerade im Winter schön sein, sich die Funde des vergangenen Frühjahrs und Sommers noch einmal vorzunehmen, sie genau zu betrachten und dann auf die Herbarbögen zu kleben. Auch das ist wieder eine gute Gelegenheit, die Namen der Pflanzen zu wiederholen und sie dabei nach und nach zu lernen.

Zum Aufkleben eignen sich gut DIN-A4-Karteikarten. Die getrockneten Pflanzen werden vorsichtig vom Einlegebogen auf den Herbarbogen übertragen. Pro Herbarbogen wird immer nur eine Art und diese auch immer nur von einem Fundort aufgeklebt. 
Die Pflanzen werden mit Streifen Nassklebeband auf die Bögen geklebt. Im Handel kann man es auf Rollen in Geschäften für Künstlerbedarf erwerben, da es in der Aquarellmalerei zum Aufkleben des Aquarellpapiers verwendet wird. Handelsübliche durchsichtige Klebestreifen sollte man nicht benutzen, da diese schnell braun werden und nur wenige Jahre ihre Klebkraft behalten. Letzteres gilt auch für Klebeetiketten.

Die Nassklebestreifen werden an unauffälliger Stelle über den Stängel oder Zweig geklebt und zwar so, dass die Pflanze sich nicht mehr verschieben lässt.

Ist die Pflanze aufgeklebt, bekommt der Herbarbogen schließlich noch ein Etikett, auf dem man den deutschen Namen, den wissenschaftlichen Namen, den Familiennamen, den Fundort und das Datum einträgt. Dieses Etikett klebt man in die linke oder rechte untere Ecke des Bogens.

\section{Schritt 5: Herbarbögen aufbewahren}

Wenn nach und nach die Sammlung der Herbarbögen wächst, stellt sich die Frage, wie man sie am besten aufbewahrt. Nach welchem System soll man sie sortieren, damit man die Pflanzen schnell wiederfindet? Und worin sollte man sie am besten aufbewahren?

Früher wurden Pflanzensammlungen ausschließlich systematisch angelegt, das heißt nach dem System eines wissenschaftlichen Bestimmungsbuches. Es gibt aber auch Sammlungen, die nach Standorten - zum Beispiel Magerrasen, Sumpf- oder Wasserpflanzen - oder nach Gift- oder Heilpflanzen angelegt werden. Nach welchem System man die Pflanzen sortiert, ist erst einmal nicht so wichtig - sie sollten nur leicht wiederzufinden sein. In den großen öffentlichen Herbarien sind die Herbarbögen heute meist alphabetisch nach den wissenschaftlichen Namen sortiert.

Herbarbögen sollten immer liegend aufbewahrt werden, denn die trockenen Pflanzen sind sehr empfindlich und brechen leicht. Unachtsames Bewegen der Belege kann in wenigen Sekunden eine ganze Reihe von Pflanzen zerstören. Deshalb wird in Herbarbögen nie wie in einem Buch geblättert. Zum Betrachten wird ein Stapel auf den Tisch gelegt und durch leichtes Anheben der Ecke, an der sich das Etikett befindet, der gesuchte Herbarbogen ermittelt. Dieser kleine, anfangs ungewohnte Handgriff garantiert, dass die Pflanzen möglichst lange halten.

Die Pflanzen sollen in einem trockenen Raum aufbewahrt werden, damit sie nicht schimmeln, zum Beispiel liegend in Schubladen, passenden Kartons oder in einem speziellen Herbarschrank. Feuchtigkeit, Staub, Licht und von außen eindringenden Insekten sind die größten Feinde der Herbarien. Bei allen drei möglichen Gefahren bieten geschlossene Schränke, Schachteln oder Schubladen einen gewissen Schutz.

\section{Schutz vor Insektenbefall}

Neben Schimmel, dem man mit einer konsequent trockenen Lagerung gut vorbeugen kann, sind Schadinsekten wie Bücherläuse die größten Feinde für ein Herbarium. Eier oder Larven, die an oder in den Pflanzen sitzen, sterben im Gegensatz zu den ausgewachsenen Tieren beim Pressen und Trocknen nicht ab. Die Larven ernähren sich vorwiegend von den zarten Pflanzenteilen. Besonders die Blüten zerstören sie oft, die dann zu Staub zerfallen. Um einen Insektenbefall zu vermeiden, sollten keine Pflanzen in die Sammlung einsortiert werden, die nicht zuvor einer insektiziden Behandlung unterzogen wurden. Denn schon ein einziger, nicht behandelter Beleg kann die ganze Sammlung schwer schädigen.

Es gibt einige ungiftige Methoden, mit denen man Herbarbögen vor Insekten schützen kann, indem man zum Beispiel

- einen Stapel von maximal $15 \mathrm{~cm}$ Dicke für mindestens 48 Stunden bei $-18^{\circ} \mathrm{C}$ einfriert,

- die Bögen in einem Trockenschrank zwei Tage bei $80^{\circ} \mathrm{C}$ erhitzt oder

- sie für ein paar Sekunden in die Mikrowelle gibt.

Um beim Einfrieren zu vermeiden, dass die Pflanzenbögen Wasser aus dem Gefrierschrank aufnehmen, sollte man sie in Plastik einwickeln.

Jeder neu hinzukommende Herbarbogen muss auf diese Weise behandelt werden, um die anderen Bögen nicht zu infizieren.

\section{Ein wertvoller Schatz}

Ein auf diese Weise so sorgfältig angelegtes Herbarium ist ein sehr wertvoller Schatz. Er kann uns nicht nur ein Leben lang Freude bereiten, er bleibt auch unserer Nachwelt jahrhundertelang erhalten.

\section{○}

$\square$ Dieser Artikel ist online zu finden: http://dx.doi.org/10.1055/a-1082-5999

\section{目 Zum Weiterlesen}

[1] Grappendorf D. Herbarium - Anleitung zum Pressen und Trocknen von Pflanzen. Alsfeld: Eigenverlag www.dorisgrappendorf.de; 2015

[2] Grappendorf D. Was blüht denn da? - Das Herbarium. Stuttgart: Kosmos; 2017

[3] Spohn M, Golte-Bechtle M, Spohn R. Was blüht denn da? Stuttgart: Kosmos; 2015

[4] Parolly G, Rohwer JG. Schmeil-Fitschen. Die Flora Deutschlands und angrenzender Länder. Heidelberg: Quelle \& Meyer; 2019

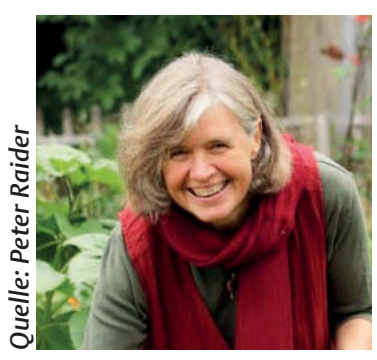

\section{HP DORIS GRAPPENDORF}

Doris Grappendorf ist Biologin, Heilpraktikerin und Pflanzenheilkundlerin (Phytotherapeutin). Sie arbeitet seit vierzig Jahren mit Heilpflanzen. Sie besitzt selbst ein Herbarium mit über 2000 Pflanzenkarten. „Meine Berufung ist es, die Menschen und die Pflanzen wieder näher zueinander zu führen“, sagt sie über sich selbst und schreibt seit einigen Jahren Bücher über Pflanzen.

\section{E-Mail: doris-grappendorf@t-online.de}

\section{Internet: www.dorisgrappendorf.de}

